\title{
BOUNDS FOR SOLUTIONS TO A CLASS OF NONLINEAR INTEGRAL EQUATIONS
}

BY

\author{
ROBERT H. MARTIN, JR.( $\left.{ }^{1}\right)$
}

\begin{abstract}
In this paper a bound is obtained for solutions to a class of nonlinear Stieltjes integral equations. The class of equations under consideration have been previously studied by J. S. Mac Nerney. It is shown that the bound obtained here is" in some sense a best bound, and in the linear case, reduces to a previous result of the author.
\end{abstract}

1. Introduction. In [6] the author obtained a bound for solutions of a class of linear integral equations which extended a result of W. A. Coppel [1, Theorem 3, p. 58] for linear differential equations. This paper obtains a similar bound for solutions of an integral equation of the form

$$
U(t)=U(a)+(R) \int_{t}^{a} d F \cdot U
$$

where the integral is the Cauchy right integral and $F$ is a function from the real numbers into a space of Lipschitz functions from a Banach space $E$ into $E$. This type of integral equation has been studied by J. W. Neuberger [7] for continuous $F$ and extended to the case where $F$ is of bounded variation by J. S. Mac Nerney [5]. It is shown that the bound obtained here is in some sense a best bound, and a necessary and sufficient condition for each solution to the above integral equation to be dominated by an exponential function is given. Also, this bound is shown to be connected with the concept of a dissipative linear operator in a Banach space as defined by G. Lumer and R. S. Phillips [3].

2. The space LIP $(E)$. Let $K$ be either the real or complex field and let $E$ be a Banach space over $K$ with norm denoted by $|\cdot|$. The class LIP $(E)$ will consist of all functions $f$ from $E$ into $E$ such that $f(0)=0$ and for which there is a constant $L$ such that $|f(x)-f(y)| \leqq L|x-y|$ for all $x$ and $y$ in $E$. With addition and scalar multiplication defined in the natural manner, LIP $(E)$ is a vector space over $K$. Furthermore, with multiplication defined by composition (i.e. if $f$ and $g$ are in LIP $(E)$, then $f \cdot g(x)=f(g(x))$ for all $x$ in $E)$, LIP $(E)$ has each of the properties of a ring with unity except left distributivity of multiplication over addition. The

Received by the editors August 19, 1969.

AMS 1969 subject classifications. Primary 3495; Secondary 3451.

Key words and phrases. Stieltjes integral equations, Banach space, bounds, Lipschitz functions, dissipative.

(1) Present address of the author is North Carolina State University at Raleigh.

Copyright 울 1971, American Mathematical Society 
identity function from $E$ into $E$ will be denoted by 1 and, when convenient, the real numbers will be considered as a subspace of LIP $(E)$.

DEFINITION 2.1. For each $f$ in LIP $(E)$ define

(i) $N[f]=\sup \{|f(x)| /|x|: x \in E, x \neq 0\}$,

(ii) $N^{\prime}[f]=\sup \{|f(x)-f(y)| /|x-y|: x, y \in E, x \neq y\}$, and

(iii) $M[f]=\lim _{h \rightarrow+0}(N[1+h f]-1) / h$.

REMARK. Both $N$ and $N^{\prime}$ are norms on LIP $(E)$ and the existence of the limit in (iii) follows, for instance, from the fact that the function $h \rightarrow N[1+h f]$ is convex.

THEOREM 2.1. If $f$ and $g$ are in LIP $(E)$ and $r>0$, then

(i) $M[r f]=r M[f]$,

(ii) $M[f+g] \leqq M[f]+M[g]$,

(iii) $|M[f]| \leqq N[f]$, and

(iv) $|M[f]-M[g]| \leqq N[f-g]$.

Indication of proof. Since $(N[1+h r f]-1) / h=r(N[1+h r f]-1) /(h r)$ and $h r \rightarrow+0$ as $h \rightarrow+0$, we have (i). Part (ii) follows from the inequality $(N[1+h(f+g)]-1) / h$ $\leqq(N[1+2 h f]-1) /(2 h)+(N[1+2 h g]-1) /(2 h)$, (iii) is immediate, and (iv) follows from (ii) and (iii).

For each $f$ in LIP $(E)$ let $\exp (f)$ denote the function $g$ from $E$ into $E$ such that $g(x)=\lim _{n \rightarrow \infty}(1+f / n)^{n} \cdot x$ for all $x$ in $E$ (where, for notational convenience, $f \cdot x=f(x)$ whenever $f$ is in LIP $(E)$ and $x$ is in $E$ ). By using Lemmas 1.1, 1.2, and 1.3 of [5], we have the following

LEMMA 2.0. If $f$ is in LIP (E) then

(i) $\exp (f)$ is an invertible member of LIP $(E)$ with $\exp (f)^{-1}=\exp (-f)$,

(ii) $\lim _{n \rightarrow \infty} N\left[\exp (f)-(1+f / n)^{n}\right]=0$, and

(iii) $N[\exp (f)-(1+f)] \leqq N^{\prime}[f]^{2} \exp \left(N^{\prime}[f]\right)$.

THEOREM 2.2. If $f$ is in LIP $(E)$ then

(i) $M[f]=\lim _{h \rightarrow+0}(N[\exp (h f)]-1) / h$,

(ii) $N[\exp (f)] \leqq \exp (M[f])$, and

(iii) $1+h M[f] \leqq N[1+h f] \leqq 1+h M[f]+h^{2} N^{\prime}[f]^{2} \exp \left(h N^{\prime}[f]\right)$ $+(\exp (h N[f])-1-h N[f])$ for all $h \geqq 0$.

Indication of proof. Part (i) is immediate from part (iii) of Lemma 2.0. If $\boldsymbol{n}$ is a positive integer, estimates of the type $N[\exp (f)] \sim N\left[(1+f / n)^{n}\right] \leqq N[1+f / n]^{n}$ $=\{1+n(N[1+f / n]-1) / n\}^{n} \sim\{1+M[f] / n\}^{n} \sim \exp (M[f])$ show that (ii) is true. The left side of (iii) holds since $(N[1+h f]-1) / h$ is nondecreasing for $h>0$. From (iii) of Lemma 2.0 and since $M[h f]=h M[f] \leqq h N[f]$, we have

$$
\begin{aligned}
N[1+h f] & \leqq N[\exp (h f)]+N[1+h f-\exp (h f)] \\
& \leqq \exp (M[h f])+N^{\prime}[h f]^{2} \exp \left(N^{\prime}[h f]\right) \\
& \leqq 1+h M[f]+\sum_{2}^{\infty} h^{n} N[f]^{n} /(n !)+h^{2} N^{\prime}[f]^{2} \exp \left(h N^{\prime}[f]\right)
\end{aligned}
$$

and (iii) follows. 
From parts (i) and (ii) of Theorem 2.2 we have

Corollary 2.1. If $f$ is in $\operatorname{LIP}(E)$, then $M[f] \leqq 0$ if and only if $N[\exp (h f)] \leqq 1$ for all $h \geqq 0$.

From part (iii) of Theorem 2.2 we have

COROLlaRY 2.2. Iff is in LIP $(E)$ and $h$ is a positive number such that $2 h N^{\prime}[f] \leqq 1$, then $|N[1+h f]-1-h M[f]| \leqq 4 h^{2} N^{\prime}[f]^{2}$.

3. Bounds for a nonlinear integral operation. Let $S$ denote the set of real numbers, let $O A^{+}$denote the set of all functions $\alpha$ from $S \times S$ into the nonnegative real numbers such that $\alpha(r, s)+\alpha(s, t)=\alpha(r, t)$ whenever $r \leqq s \leqq t$ or $r \geqq s \geqq t$, and let $O M^{+}$denote the set of all functions $\mu$ from $S \times S$ into the real numbers not less than one such that $\mu(r, s) \cdot \mu(s, t)=\mu(r, t)$ whenever $r \leqq s \leqq t$ or $r \geqq s \geqq t$. The class $O A$ (see [5, p. 622]) will consist of all functions $V$ from $S \times S$ into LIP $(E)$ such that

1. there is an $\alpha$ in $O A^{+}$such that $N^{\prime}[V] \leqq \alpha$, and

2. $V(r, s)+V(s, t)=V(r, t)$ whenever $r \leqq s \leqq t$ or $r \geqq s \geqq t$.

The class $O M$ (see [5, p. 622]) will consist of all functions $W$ from $S \times S$ into LIP $(E)$ such that

1. there is a $\mu$ in $O M^{+}$such that $N^{\prime}[W-1] \leqq \mu-1$, and

2. $W(r, s) \cdot W(s, t)=W(r, t)$ whenever $r \leqq s \leqq t$ or $r \geqq s \geqq t$.

Considering the space of real numbers as a subspace of LIP $(E)$, we will let $O A^{r}$ (respectively $O M^{r}$ ) denote the set of all members of $O A$ (respectively $O M$ ) which are real valued.

If $(a, b)$ is in $S \times S$ then $\left(u_{p}\right)_{0}^{n}$ is a subdivision of $(a, b)$ if $u_{0}=a, u_{n}=b$, and $u_{p-1} \leqq u_{p}$ or $u_{p-1} \geqq u_{p}$ according to whether $a \leqq b$ or $a>b$. The continuously continued sums and products are defined in [5, p. 624] and we indicate their definitions here with their approximations (the limits are taken in the sense of refinements of subdivisions).

1. If $g$ is a function from $S \times S$ into $E$, then ${ }_{a} \sum^{b} g \sim \sum_{u} g=\sum_{1}^{n} g\left(u_{p-1}, u_{p}\right)$.

2. If $H$ is a function from $S \times S$ into $\operatorname{LIP}(E)$ and $x$ is in $E$, then

$$
\left(\prod_{a}^{b} H\right) \cdot x \sim\left(\prod_{u} H\right) \cdot x=H\left(u_{0}, u_{1}\right) \cdots H\left(u_{n-1}, u_{n}\right) \cdot x .
$$

Also, ${ }_{a} \Pi^{b} H$ denotes a member $f$ of $\operatorname{LIP}(E)$ such that $f(x)=\left({ }_{a} \Pi^{b} H\right) \cdot x$ for all $x$ in $E$.

3. If $V$ is a function from $S \times S$ into $\operatorname{LIP}(E)$ and $U$ is a function from $S$ into $E$, then $(R)_{a} \int^{b} V \cdot U={ }_{a} \sum^{b} g$ where $g(s, t)=V(s, t) \cdot U(t)$ for all $(s, t)$ in $S \times S$.

In [4, Theorem 2.2] Mac Nerney shows that there is a bijection $\mathscr{E}^{+}$from $O A^{+}$ into $O M^{+}$such that if $\mathscr{E}^{+}[\alpha]=\mu$, then

1. $\mu(a, b)={ }_{a} \Pi^{b}(1+\alpha)$ for all $(a, b)$ in $S \times S$, and

2. $\alpha(a, b)={ }_{a} \sum^{b}(\mu-1)$ for all $(a, b)$ in $S \times S$. 
In [5, Theorem 1.1] Mac Nerney shows that there is a bijection $\mathscr{E}$ from $O A$ into $O M$ such that if $\mathscr{E}[V]=W$, then

1. $W(a, b)={ }_{a} \Pi^{b}(1+V)$ for all $(a, b)$ in $S \times S$,

2. $V(a, b)={ }_{a} \sum^{b}(W-1)$ for all $(a, b)$ in $S \times S$, and

3. there is an $\alpha$ in $O A^{+}$such that if $\mu=\mathscr{E}^{+}[\alpha]$, then $N[W(a, b)-1-V(a, b)]$ $\leqq \mu(a, b)-1-\alpha(a, b)$ for all $(a, b)$ in $S \times S$.

Furthermore, (see [5, Theorem 2]) if $V$ is in $O A, c$ is in $S$, and $U$ is a function from $S$ into $E$ which is of bounded variation on bounded subintervals of $S$, then these are equivalent:

1. $U(t)=\mathscr{E}[V](t, c) \cdot U(c)$ for all $t$ in $S$.

2. $U(t)=U(c)+(R)_{t} \int^{c} V \cdot U$ for all $t$ in $S$.

LEMMA 3.1. Suppose that $V$ is in $O A, \alpha$ is in $O A^{+}$such that $N^{\prime}[V] \leqq \alpha, W$ is in $O M, \mu$ is in $O M^{+}$such that $N^{\prime}[W-1] \leqq \mu-1$, and $(a, b)$ is in $S \times S$. Then whenever $u$ is a subdivision of $(a, b)$ and $v$ is a refinement of $u$,

(i) $\sum_{u} M[V] \leqq \sum_{v} M[V] \leqq \alpha(a, b)$ so that ${ }_{a} \sum^{b} M[V]$ exists, and

(ii) $\prod_{u} N[W] \leqq \prod_{v} N[W] \leqq \mu(a, b)$ so that ${ }_{a} \Pi^{b} N[W]$ exists.

LemMA 3.2. Suppose that $V$ is in $O A, \alpha$ is in $O A^{+}$such that $N^{\prime}[V] \leqq \alpha$, and $(a, b)$ is in $S \times S$. Then

(i) if there is a subdivision $\left(u_{p}\right)_{0}^{n}$ of $(a, b)$ such that $\alpha\left(u_{p-1}, u_{p}\right) \leqq \delta \leqq 1 / 2$ for each $1 \leqq p \leqq n$, then $\left|\sum_{u}(N[1+V]-1)-\sum_{u} M[V]\right| \leqq 4 \delta \alpha(a, b)$;

(ii) for each $\delta>0$ there is a subdivision $\left(v_{p}\right)_{0}^{n}$ of $(a, b)$ such that if $v_{p-1}<s \leqq t<v_{p}$ or $v_{p-1}>s \geqq t>v_{p}$, then $\alpha(s, t) \leqq \delta$; and

(iii) for each $s$ in $S$ and each $\varepsilon>0$, there is a $\delta>0$ such that if $s<t \leqq s+\delta$ then $\alpha(t, s+\delta) \leqq \varepsilon$ and if $s>t \geqq s-\delta$ then $\alpha(s-\delta, t) \leqq \varepsilon$.

Indication of proof. By using Corollary 2.2, $\left|\sum_{u}(N[1+V]-1)-\sum_{u} M[V]\right|$ $\leqq \sum_{u} 4 N^{\prime}[V]^{2}$ and (i) follows. Both (ii) and (iii) are true since $\alpha$ can be uniformly approximated by step functions.

LemmA 3.3. If $V$ is in $O A$ and $(a, b)$ is in $S \times S$, then ${ }_{a} \sum^{b}(N[1+V]-1)$ exists.

By using Lemmas 3.1 and 3.2 the proof is essentially the same as in the linear case (see [6, Theorem 1]) and is omitted.

THEOREM 3.1. Suppose that $V$ is in $O A, W=\mathscr{E}[V]$, and for each $(a, b)$ in $S \times S$, $\sigma[V](a, b)={ }_{a} \sum^{b}(N[1+V]-1)$ and $\pi[W](a, b)={ }_{a} \Pi^{b} N[W]$. Then

(i) $\sigma[V]$ is in $O A^{r}$ and if $\alpha$ is in $O A^{+}$such that $N^{\prime}[V] \leqq \alpha$, then $|\sigma[V]| \leqq \alpha$.

(ii) $\pi[W]$ is in $O M^{r}$ and if $\mu$ is in $O M^{+}$such that $N^{\prime}[W-1] \leqq \mu-1$, then $|\pi[W]-1| \leqq \mu-1$.

(iii) $\pi[W]=\mathscr{E}[\sigma[V]]$.

Indication of proof. $\sigma[V]$ is clearly order-additive and real valued. The inequalities $|N[1+V]-1| \leqq N[V] \leqq N^{\prime}[V]$ show that (i) is true. $\pi[W]$ is clearly order-multiplicative and real valued. Suppose that $(a, b)$ is in $S \times S, \mu=\mathscr{E}^{+}[\alpha]$, and 
$\left(u_{p}\right)_{0}^{n}$ is a subdivision of $(a, b)$. By using property 3 in the definition of $\mathscr{E}$, we have that

$$
\begin{array}{rl}
\mid \prod_{u} & N[W]-\prod_{u} N[1+V] \mid \\
= & \sum_{p=1}^{n}\left\{\prod_{1}^{p-1} N\left[1+V\left(u_{i-1}, u_{i}\right)\right]\right\} \cdot\left\{N\left[W\left(u_{p-1}, u_{p}\right)\right]-N\left[1+V\left(u_{p-1}, u_{p}\right)\right]\right\} \\
& \leqq \prod_{u}(\mu) \cdot \cdot \sum_{1}^{n} \mid N\left[W\left(u_{p-1}^{n} N\left[W\left(u_{i-1}, u_{i}\right)\right]\right\} \mid\right. \\
& \leqq \mu(a, b) \cdot\left\{\sum_{1}^{n}\left[\mu\left(u_{p-1}, u_{p}\right)-1\right]-\alpha(a, b)\right\} .
\end{array}
$$

Consequently, by property 2 of the definition of $\mathscr{E}^{+},{ }_{a} \Pi^{b} N[1+V]={ }_{a} \Pi^{b} N[W]$. Suppose that $\varepsilon$ is a positive number. Then there is a subdivision $\left(u_{p}\right)_{0}^{n}$ of $(a, b)$ such that $\sum_{1}^{n}\left|\sigma[V]\left(u_{p-1}, u_{p}\right)-\left(N\left[1+V\left(u_{p-1}, u_{p}\right)\right]-1\right)\right| \leqq \varepsilon$. Thus,

$$
\begin{aligned}
\left|\prod_{1}^{n}\left(1+\sigma[V]\left(u_{p-1}, u_{p}\right)\right)-\prod_{1}^{n} N\left[1+V\left(u_{p-1}, u_{p}\right)\right]\right| \\
\quad \leqq\left\{\prod_{1}^{n}\left[1+\alpha\left(u_{p-1}, u_{p}\right)\right]\right\} \cdot\left\{\sum_{1}^{n} \mid 1+\sigma[V]\left(u_{p-1}, u_{p}\right)-N\left[1+V\left(u_{p-1}, u_{p}\right)\right]\right\} \\
\leqq \mu(a, b) \cdot \varepsilon .
\end{aligned}
$$

Consequently, ${ }_{a} \Pi^{b} N[W]={ }_{a} \Pi^{b} N[1+V]={ }_{a} \Pi^{b}(1+\sigma[V])=\mathscr{E}[\sigma[V]](a, b)$ and both (ii) and (iii) are true.

As an immediate consequence of Theorem 3.1 we have

THEOREM 3.2. Suppose that $V$ is in $O A$, $a$ is in $S, U$ is a function from $S$ into $E$ which is of bounded variation on bounded subintervals of $S$, and for each $t$ in $S$,

$$
U(t)=U(a)+(R) \int_{t}^{a} V \cdot U .
$$

Then $|U| \leqq \psi$ where, for each $t$ in $S$,

$$
\psi(t)=|U(a)|+(R) \int_{t}^{a} \sigma[V] \cdot \psi
$$

\{i.e. $|U(t)|=|\mathscr{E}[V](t, a) \cdot U(a)| \leqq \mathscr{E}[\sigma[V]](t, a) \cdot|U(a)|=\psi(t)\}$.

Example 3.1. Suppose that $F$ is a function from $S$ into LIP $(E)$ which is continuous for the norm $N^{\prime}$. For each $(s, t)$ in $S \times S$ define the member $V$ of $O A$ by $V(s, t)={ }_{s} \int^{t} F(r) d r$ if $s \leqq t$ and $V(s, t)=V(t, s)$ if $s \geqq t$. If $a$ is in $S$ and $x$ is in $E$, the function $U$ from $[a, \infty)$ into $E$ defined by $U(t)=\mathscr{E}[V](t, a) \cdot x$ is continuously 
differentiable and satisfies $U^{\prime}(t)=F(t) \cdot U(t)$. If $(s, t)$ is in $S \times S, s \leqq t$, and $\left(u_{p}\right)_{0}^{n}$ is a subdivision of $(s, t)$, then estimates of the type

$$
\begin{aligned}
\sum_{1}^{n}\left(N\left[1+\int_{u_{p-1}}^{u_{p}} F(r) d r\right]-1\right) & \sim \sum_{1}^{n}\left\{\frac{N\left[1+\left(u_{p}-u_{p-1}\right) F\left(u_{p}\right)\right]-1}{\left(u_{p}-u_{p-1}\right)}\right\} \cdot\left(u_{p}-u_{p-1}\right) \\
& \sim \sum_{1}^{n} M\left[F\left(u_{p}\right)\right]\left(u_{p}-u_{p-1}\right)
\end{aligned}
$$

show that $\sigma[V](s, t)={ }_{s} \int^{t} M[F(r)] d r$. Similarly, if $s \geqq t, \sigma[V](s, t)=\sigma[V](t, s)$. Thus, if $\psi(t)=\mathscr{E}[\sigma[V]](t, a) \cdot|U(a)|$ for all $t$ in $[a, \infty)$, then $\psi$ is continuously differentiable and satisfies $\psi^{\prime}(t)=M[F(t)] \psi(t)$. Hence, if $t$ is in $[a, \infty)$,

$$
|U(t)| \leqq \psi(t)=|U(a)| \exp \left(\int_{a}^{t} M[F(r)] d r\right) .
$$

If $F(t)$ is a linear member of LIP $(E)$ for all $t$ in $S$, then this is the bound obtained by Coppel [1, Theorem 3, p. 58].

We also have that $\mathscr{E}[\sigma[V]]$ is a "best" bound for $\mathscr{E}[V]$ in the following sense:

THEOREM 3.3. Suppose that $V$ is in $O A, \lambda$ is in $O A^{r}$, and $N[\mathscr{E}[V]](a, b) \leqq \mathscr{E}[\lambda](a, b)$ for all $(a, b)$ in $S \times S$. Then $\sigma[V](a, b) \leqq \lambda(a, b)$-and hence $\mathscr{E}[\sigma[V]](a, b) \leqq \mathscr{E}[\lambda](a, b)$ - for all $(a, b)$ in $S \times S$.

Indication of proof. Since $\pi[\mathscr{E}[V]] \leqq \mathscr{E}[\lambda]$ we have by part (iii) of Theorem 3.1, that, for each $(a, b)$ in $S \times S$,

$$
\sigma[V](a, b)={ }_{a} \sum^{b}(\pi[\mathscr{E}[V]]-1) \leqq{ }_{a} \sum^{b}(\mathscr{E}[\lambda]-1)=\lambda(a, b)
$$

and the theorem is true.

REMARK. The corresponding result to Theorem 3.3 in the differential equation setting of Example 3.1 is that the function $t \rightarrow M[F(t)]$ is the smallest continuous function $g$ from $S$ into the real numbers such that $|U(t)| \leqq|U(a)| \exp \left({ }_{a}{ }^{t} g(r) d r\right)$ for all $a$ in $S, t$ in $[a, \infty)$, and all differentiable functions $U$ from $[a, \infty)$ into $E$ such that $U^{\prime}(t)=F(t) \cdot U(t)$.

COROLlaRY 3.1. With the supposition of Theorem 3.2, these are equivalent:

(i) There is a number $c$ such that $\sigma[V](s, t) \leqq c|t-s|$ for all $(s, t)$ in $S \times S$.

(ii) There is a number $c$ such that whenever $a$ is in $S$ and

$$
U(t)=U(a)+(R) \int_{t}^{a} V \cdot U
$$

for all $t$ in $S$, then $|U(t)| \leqq|U(a)| \exp (c|t-a|)$ for all $t$ in $S$.

Indication of proof. If (i) holds then (ii) is immediate from Theorem 3.2. If (ii) holds, then $|\mathscr{E}[V](t, a) \cdot x| \leqq \exp (c|t-a|)|x|$ for all $x$ in $E$ and all $(t, a)$ in $S \times S$ so that $N[\mathscr{E}[V](t, a)] \leqq \exp (c|t-a|)$. Hence, (i) follows from Theorem 3.3 with $\lambda(s, t)=c|t-s|$ for all $(s, t)$ in $S \times S$. 
4. Dissipative members of $O A$. Let $O A D$ denote the set of all $V$ in $O A$ such that $\sigma[V] \leqq 0$ and let $O M D$ denote the set of all $W$ in $O M$ such that $\pi[W] \leqq 1$. It is immediate that a member $W$ of $O M$ is in $O M D$ if and only if $N[W] \leqq 1$. Furthermore, it follows from part (iii) of Theorem 3.1 that $\mathscr{E}$ maps $O A D$ onto $O M D$.

Definition 4.1. A member $f$ of $\operatorname{LIP}(E)$ will be called dissipative if and only if $M[f] \leqq 0$.

REMARK. In [3] Lumer and Phillips define dissipative linear operators in a Banach space. Let $E^{\prime}$ denote the dual of $E$ and for each $x$ in $E$ let $x^{\prime}$ be a member of $E^{\prime}$ such that $x^{\prime}(x)=|x|^{2}$. If for each $x$ and $y$ in $E,[x, y]=y^{\prime}(x)$, then $[x, y]$ is a semi-inner-product on $E$ (see [2, Definition 1 and Theorem 2]). If $f$ is a linear member of $\operatorname{LIP}(E)$ it is called dissipative if $\operatorname{Re}[f(x), x] \leqq 0$ for all $x$ in $E$ (see [3, p. 679]). Lumer and Phillips show this to be equivalent to $M[f] \leqq 0$. This is also the case for any $f$ in LIP $(E)$ but is not needed here.

THEOREM 4.1. If $F$ and $V$ are as in Example 3.1, then these are equivalent:

(i) $V$ is in $O A D$.

(ii) $M[F(t)] \leqq 0$ for all $t$ in $S$.

(iii) Each differentiable function $U$ from $S$ into $E$ such that $U^{\prime}(t)=F(t) \cdot U(t)$ is nonincreasing in norm (i.e. the function $t \rightarrow|U(t)|$ of $S$ into the real numbers is nonincreasing).

Indication of proof. Since $\sigma[V](s, t)={ }_{s} \int^{t} M[F(r)] d r$ if $s \leqq t$ and $\sigma[V](s, t)$ $=\sigma[V](t, s)$ if $s \geqq t, \sigma[V](s, t) \leqq 0$ for all $(s, t)$ in $S \times S$ if and only if $M[F(t)] \leqq 0$ for all $t$ in $S$. Thus, (i) and (ii) are equivalent. Whenever $a \leqq t$ we have that $|U(t)|$ $\leqq|U(a)| \exp \left({ }_{a} \int^{t} M[F(r)] d r\right)$ so that (ii) implies (iii).

The remark following Theorem 3.3 shows that (iii) implies (ii).

Let $H$ denote the class of all functions from $E$ into $E$ which map 0 into 0 and let $V^{*}$ be a function from $S \times S$ into $H$ with the property that for each positive integer $n$ there is a member $V_{n}$ of $O A D$ such that $V^{*}(s, t) \cdot x=V_{n}(s, t) \cdot x$ for all $(s, t)$ in $S \times S$ and all $x$ in $E$ with $|x|<n$.

Lemma 4.1. Suppose that $V^{*}$ and $\left(V_{n}\right)_{1}^{\infty}$ are as above, $x$ is in $E$, and $(a, b)$ is in $S \times S$. Then $\left({ }_{a} \Pi^{b}\left[1+V^{*}\right]\right) \cdot x$ exists and if $n>|x|$, then $\left({ }_{a} \Pi^{b}\left[1+V^{*}\right]\right) \cdot x$ $=\mathscr{E}\left[V_{n}\right](a, b) \cdot x$.

Indication of proof. Suppose that $n>|x|$ and $\varepsilon$ is a positive number such that $n>(1+\varepsilon)|x|$. Since ${ }_{a} \prod^{b} N\left[1+V_{n}\right]=\mathscr{E}\left[\sigma\left[V_{n}\right]\right](a, b) \leqq 1$ (see the proof of Theorem $3.1)$, there is a subdivision $v$ of $(a, b)$ such that if $\left(u_{p}\right)_{0}^{m}$ is a refinement of $v$, then

$$
\left|\left\{\prod_{i=j}^{k}\left[1+V_{n}\left(u_{i-1}, u_{i}\right)\right]\right\} \cdot x\right| \leqq\left\{\prod_{i=j}^{k} N\left[1+V_{n}\left(u_{i-1}, u_{i}\right)\right]\right\} \cdot|x| \leqq(1+\varepsilon)|x|<n
$$

whenever $1 \leqq j \leqq k \leqq m$. Thus, whenever $u$ is a refinement of $v,\left(\prod_{u}\left[1+V^{*}\right]\right) \cdot x$ $=\left(\prod_{u}\left[1+V_{n}\right]\right) \cdot x$ and the lemma follows. 
THEOREM 4.2. Suppose that $V^{*}$ and $\left(V_{n}\right)_{1}^{\infty}$ are as in Lemma 4.1, a is in $S$, and $U$ is a function from $S$ into $E$ which is of bounded variation on bounded subintervals of $S$. Then these are equivalent:

(i) $U(t)=U(a)+(R)_{t} \int^{a} V^{*} \cdot U$ for all $t$ in $S$.

(ii) $U(t)=\left({ }_{t} \Pi^{a}\left[1+V^{*}\right]\right) \cdot U(a)$ for all $t$ in $S$.

Indication of proof. Suppose that (ii) is true. By Lemma 4.1 we have $U(t)$ $=\mathscr{E}\left[V_{n}\right](t, a) \cdot U(a)$ whenever $n>|U(a)|$ and $t$ is in $S$. Since $V_{n}$ is in $O A D,|U(t)|$ $\leqq|U(a)|<n$ for all $t$ in $S$ so that if $u$ is a subdivision of $(t, a)$, then $V^{*}\left(u_{p-1}, u_{p}\right)$ . $U\left(u_{p}\right)=V_{n}\left(u_{p-1}, u_{p}\right) \cdot U\left(u_{p}\right)$ and (i) follows.

Now suppose (i) is true and that $t$ is in $S$. Then there is an integer $n$ such that $|U(s)|<n$ for all $s$ such that $a \leqq s \leqq t$ or $a \geqq s \geqq t$. Thus $(R)_{s} \int^{a} V^{*} \cdot U=(R)_{s} \int^{a} V_{n} \cdot U$ for all such $s$ and we have that $U(t)=\mathscr{E}\left[V_{n}\right](t, a) \cdot U(a)$. Part (ii) now follows.

EXAMPLE 4.1. Suppose that $E$ is the space of real numbers. For each $x$ in $E$ let $g(x)=-x^{3}$ and define $V^{*}(s, t)=|t-s| g$ for all $(s, t)$ in $S \times S$. For each positive integer $n$ and each $(s, t)$ in $S \times S$ define $V_{n}(s, t)=|t-s| f_{n}$ where $f_{n}(x)=g(x)$ if $|x|<n$ and $f_{n}(x)=g(n x /|x|)$ if $|x| \geqq n$. A routine computation shows that $M\left[f_{n}\right]=0$ for all $n$ and hence, $V_{n}$ is in $O A D$ for all $n$. Thus, if $a$ is in $S, x$ is in $E$, and $U$ is the function from $[a, \infty)$ into $E$ defined by $U(t)=\left({ }_{t} \Pi^{a}\left[1+V^{*}\right]\right) \cdot x$, then $U(a)=x$ and $U^{\prime}(t)$ $=-[U(t)]^{3}$ for all $t$ in $[a, \infty)$ i.e. $\left({ }_{t} \Pi^{a}\left[1+V^{*}\right]\right) \cdot x=x\left[2 x^{2}(t-a)+1\right]^{-1 / 2}$ for all $t$ in $[a, \infty)$ and $x$ in $E\}$.

\section{REFERENCES}

1. W. A. Coppel, Stability and asymptotic behavior of differential equations, Heath, Boston, Mass., 1965. MR 32 \#7875.

2. G. Lumer, Semi-inner-product spaces, Trans. Amer. Math. Soc. 100 (1961), 29-43. MR 24 \#A2860.

3. G. Lumer and R. S. Phillips, Dissipative operators in a Banach space, Pacific J. Math. 11 (1961), 679-698. MR 24 \#A2248.

4. J. S. Mac Nerney, Integral equations and semigroups, Illinois J. Math. 7 (1963), 148-173. MR 26 \#1726.

5. - A nonlinear integral operation, Illinois J. Math. 8 (1964), 621-638. MR 29 \#5082.

6. R. H. Martin, Jr., A bound for solutions of Volterra-Stieltjes integral equations, Proc. Amer. Math. Soc. 23 (1969), 506-512. MR 40 \#662.

7. J. W. Neuberger, Continuous products and nonlinear integral equations, Pacific J. Math. 8 (1958), 529-549. MR 21 \#1509.

Georgia Institute of Technology, Atlanta, Georgia 30332 